\title{
On passive emitter tracking in sensor networks
}

\author{
Regina Kaune \\ Fraunhofer FKIE \\ Germany \\ Darko Mušicki \\ Hanyang University \\ Korea \\ Wolfgang Koch \\ Fraunhofer FKIE \\ Germany
}

\section{Introduction}

Many applications require fast and accurate localization and tracking of non-cooperative emitters. In many cases, it is advantageous not to conceal the observation process by using active sensors, but to work covertly with passive sensors. The estimation of the emitter state is based on various types of passive measurements by exploiting signals emitted by the targets. In other applications there is no choice but to exploit received signals only. Typical examples include search and rescue type operations.

Some passive measurements can be taken by single sensors: e.g. bearing measurements (AOA: Angle of Arrival) and frequency measurements (FOA: Frequency of Arrival). The emitter state can be estimated based on a set of measurements of a single passive observer. This problem is called the Target Motion Analysis (TMA) problem which means the process of estimating the state of a radiating target from noisy incomplete measurements collected by one or more passive observer(s). The TMA problem includes localization of stationary as well as tracking of moving emitters. The TMA problem based on a combination of AOA and FOA measurements is considered by Becker in (Becker, 2001). Becker investigates and discusses the TMA problem with many characteristic features such as observability conditions, combination of various types of measurements, etc., (Becker, 1999; 2005) .

Alternatively, measurements can be obtained from a network of several spatially dislocated sensors. Here, a minimum of two sensors is often needed. Measurements of Time Difference of Arrival (TDOA) and Frequency Difference of Arrival (FDOA) belong to this group.

TDOA measurements are obtained in the following way: several distributed, time-synchronized sensors measure the Time of Arrival (TOA) of signals transmitted from the emitter. The difference between two TOA measurements of the same signal gives one TDOA measurement. Alternatively, TDOA measurements can be obtained by correlating signals received by the sensors. A time standard can be used for time synchronization. 
In the absence of noise and interference, a single TDOA measurement localizes the emitter on a hyperboloid with the two sensors as foci. By taking additional independent TDOA measurements from at least four sensors, the three-dimensional emitter location is estimated from the intersections of three or more hyperboloids. If sensors and emitter lie in the same plane, one TDOA measurement defines a hyperbola describing possible emitter locations. Therefore, the localization using TDOA measurements is called hyperbolic positioning. The sign of the measurement defines the branch of the hyperbola on which the emitter is located. The two-dimensional emitter location is found at the intersection of two or more hyperbolae from at least three sensors. This intersection point can be calculated by analytical solution, see e.g. (K. C. Ho, 2008; So et al., 2008). Alternatively, a pair of two sensors moving along arbitrary but known trajectories can be used for localizing an emitter using TDOA measurements. In this case, the emitter location can be estimated by filtering and tracking methods based on further measurements over time. This chapter is focused on the localization of unknown, non-cooperative emitters using TDOA measurements from a sensor pair. Some results have already been published in (Kaune, 2009).

The localization and tracking a non-cooperative emitter can be improved by combining different kinds of passive measurements, particularly in the case of a moving emitter.

One possibility is based on bearing measurements. A pair of one azimuth and one TDOA measurement is processed at each time step. The additional AOA measurement can solve the ambiguities appearing in processing TDOA measurements only. Another possibility considers two sensors measuring the FDOA between two frequencies of arrival (Mušicki et al., 2010; Mušicki \& Koch, 2008). These measurements can be taken by the same sensors as the TDOA measurements. The TDOA/FDOA measurement pairs can be obtained by using the Complex Ambiguity function (CAF). The combination of TDOA and FDOA measurements improves the estimation performance strongly.

This chapter gives an overview of the topic of passive emitter tracking. Section 2 describes the situation of a single passive observer. Important steps of solving the passive emitter tracking problems are presented. When assessing an estimation task, it is important to know the best estimation accuracy that can be obtained with the measurements. The Cramér Rao Lower Bound (CRLB) provides a lower bound on the estimation accuracy for any unbiased estimator and reveals characteristic features of the estimation problem.

Powerful estimation algorithms must be applied to obtain useful estimates of the emitter state. For passive emitter tracking, measurements and states are not linearly related. Therefore, only nonlinear estimation methods are appropriate. Passive emitter tracking is a complex problem. Depending on the types of measurements, various estimation methods can be applied showing different localization performance in various scenarios. The goal of this chapter is to provide a review of the state of the art. The discussion is not restricted to one chosen method but presents an overview of different methods. The algorithms are not shown in detail; therefore, a look at the references is necessary to implement them. In the appendix, a toolbox of methods makes several estimation methods available which are applied in this chapter. Firstly, the maximum likelihood estimator (MLE) as a direct search method, which evaluates at each estimate the complete measurement dataset. Secondly, Kalman filter based solutions which recursively update the emitter state estimates. The tracking problem is nonlinear; thus the Extended Kalman Filter (EKF) provides an analytic approximation, while the Unscented Kalman Filter (UKF) deterministically selects a small number of points and transforms these points nonlinearly. Thirdly, Gaussian Mixture (GM) filters will be discussed, which approxi- 
mate the posterior density by a GM (a weighted sum of Gaussian density functions). Additionally, some basics on the CRLB and the Normalized Estimation Error Squared (NEES) are presented.

In sections 3, 4, 5 passive emitter tracking using TDOA, a combination of TDOA and AOA and a combination of TDOA and FDOA is investigated, respectively. Finally, conclusions are drawn.

\section{Review of TMA techniques}

Passive emitter tracking using a single passive observer is part of the TMA problem which addresses the process of estimating the state of an emitter from noisy, incomplete measurements collected by a passive observer (Becker, 1999; 2001; 2005, and references cited therein). Typical applications can be found in passive sonar, infrared (IR), or passive radar tracking systems.

\subsection{Solution of the TMA problem}

The TMA problem is solved in three consecutive steps:

- The first step is the calculation and analysis of the CRLB. It is a lower bound for the achievable estimation accuracy and reveals characteristic features of the TMA problem under consideration.

- The main step is the development of an algorithm that effectively estimates the target state from the noisy measurements collected by the observer.

- A final third step is necessary in the TMA solution process. It increases the estimation accuracy by observer motions.

These three steps can be applied to passive emitter tracking in sensor networks as well, while the third step is not as important as in the single observer case.

In the following, the solution of the TMA problem is analyzed in detail:

In evaluating an estimation problem, it is important to know the optimal estimation accuracy achievable from the measurements. It is well known that the CRLB provides a lower bound on the achievable estimation accuracy; for explicit formulas see A.1. The investigation of the CRLB provides insight into the parametric dependencies of the TMA problem under consideration. It reveals characteristic features of the localization and tracking process. For the two-dimensional TMA problem based on AOA and FOA measurements, it has been discussed in detail, (Becker, 1992). It proved that the orientation of the error ellipses of bearings and frequency measurements significantly differ. One bearing measurement provides a strip of infinite length in the position space and two frequency measurements give a strip of infinite length in the position space, too. The error ellipses of the bearing and the frequency measurements are rotated with respect to each other. Therefore, there is a gain in accuracy by combining angle and frequency measurements in the TMA situation.

The main step of the TMA problem is the development of an algorithm that effectively estimates the emitter state from noisy measurements collected by the observer. These algorithms require the modeling of the emitter dynamics and the measurement process. The system or dynamics model describes the evolution of the emitter state with time. Let $\mathbf{e}_{k} \in \mathbb{R}^{n_{e}}$ be the emitter state at time $t_{k}$, where $n_{e}$ is the dimension of the state vector, involving position and velocity. Using the evolution function $f$, the emitter state can be modeled from the previous time step $t_{k-1}$ by adding white Gaussian noise; we obtain the dynamic model:

$$
\mathbf{e}_{k}=f\left(\mathbf{e}_{k-1}\right)+\mathbf{v}_{k}, \quad \mathbf{v}_{k} \sim \mathcal{N}(\mathbf{0}, \mathbf{Q}),
$$


where $\mathbf{v}_{k} \sim \mathcal{N}(\mathbf{0}, \mathbf{Q})$ means that $\mathbf{v}_{k}$ is zero-mean normal distributed with covariance $\mathbf{Q}$. The measurement model relates the noisy measurements $\mathbf{z}_{k} \in \mathbb{R}^{n_{z}}$ to the state, where $n_{z}$ is the dimension of the measurement vector. The measurement function $h(\mathbf{e})$ is a function of the emitter state, nonlinear or linear, and reflects the relations between the emitter state and the measurements. Thus, the measurement process is modeled by adding white Gaussian noise $\mathbf{u}_{k}$ :

$$
\mathbf{z}_{k}=h\left(\mathbf{e}_{k}\right)+\mathbf{u}_{k}, \quad \mathbf{u}_{k} \sim \mathcal{N}(\mathbf{0}, \mathbf{R}),
$$

where $\mathbf{R}$ is the covariance of the measurement noise.

An estimation algorithm must be found to solve the emitter tracking problem. Based on all available measurements $Z_{k}=\left\{\mathbf{z}_{1}, \mathbf{z}_{2}, \ldots, \mathbf{z}_{k}\right\}$ up to time $t_{k}$ we seek to estimate the emitter state $\mathbf{e}_{k}$. Therefore, it is required to compute the posterior probability density function $p\left(\mathbf{e}_{k} \mid Z_{k}\right)$. A short review of available estimation algorithms is given in A.3 and include:

- As a direct method, maximum likelihood estimation (MLE) evaluates at each time step the complete measurement dataset. In many cases, a numerical iterative search algorithm is needed to implement MLE.

- Recursive Kalman-type filter algorithms can be used as well. They are Bayesian estimators and construct the posterior density using the Bayes rule. Since the measurement equation in passive emitter tracking is often nonlinear, nonlinear versions of it must be used: the Extended Kalman filter (EKF) provides an analytic approximation, while the Unscented Kalman filter (UKF) deterministically selects a small number of points and transforms these points according to the nonlinearity.

- Gaussian Mixture (GM) filters approximate the required densities by Gaussian Mixtures, weighted sums of Gaussians. The approximation can be made as accurate as desirable by adapting the number of mixture components appropriately, see (Ristic et al., 2004).

In passive tracking, the emitter may not be observable from available measurements in some situations. If the observer is moving directly in direction of the stationary emitter, for example, the emitter is not observable from bearing measurements only. In the literature, necessary and sufficient observability criteria using angle measurements and using a combination of angle and frequency measurements have been derived (Becker, 1993; 1996). In general, ambiguities can be resolved by suitable observer maneuvers, which depend on the type of measurements and the emitter model as well. A measurement set consisting of different measurement types often results in less restrictive observability conditions.

In an application, the user should always strive to get the maximum of attainable estimation accuracy. Estimation accuracy can firstly be influenced by the choice of the estimation algorithm and, secondly, by the choice of the emitter-observer geometry over time, via observer motion. The estimation accuracy highly depends on the emitter-observer geometry. The emitter-observer geometry may be changed by observer maneuvers. Thus, the final step in solving the TMA problem is to find an optimal observer maneuver creating a geometry that maximizes the estimation accuracy. In the literature, several criteria have been used, one of them is maximizing the determinant of the Fisher Information Matrix (FIM) J.

\subsection{TMA based on bearing and frequency measurements}

The standard TMA method is based on bearing measurements taken at different points along the sensor trajectory, see Figure 1. It has been the topic of much research in the literature. 
Already a single bearing measurement provides information on the emitter position. In addition, or instead of bearing measurements, measurements of the Doppler-shifted frequency can be taken, (Becker, 1992). Frequency measurements depend on the emitter-sensor-motion, more precisely on the radial component of the relative velocity vector. Frequency drift and frequency hopping have an impact on the quality of frequency measurements and have to be taken into account. The location methods based on bearing or frequency measurements differ significantly. The substantial differences between both methods lead to a significant integration gain when the combined set of bearing and frequency measurements is processed.

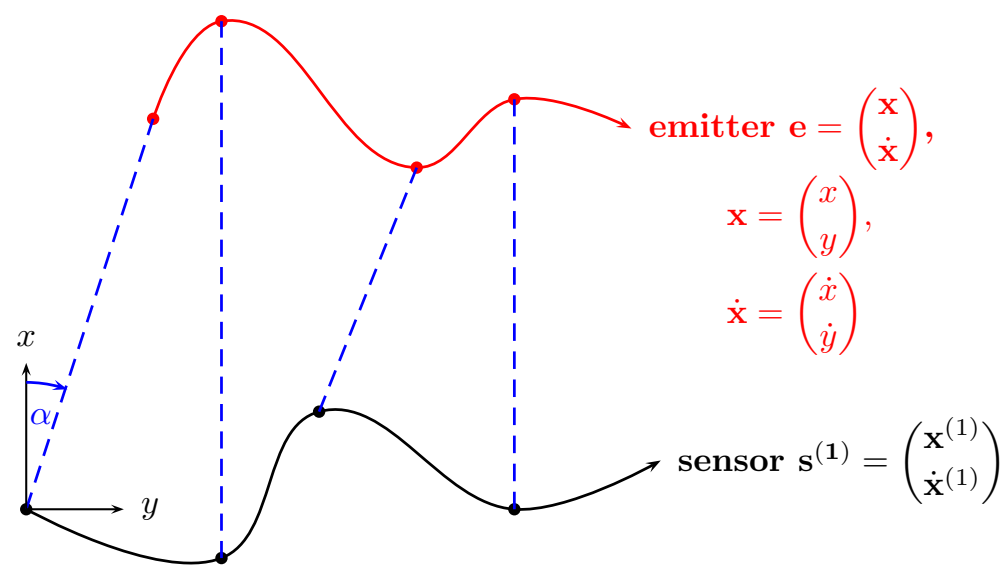

Fig. 1. TMA problem based on azimuth measurements (dashed lines)

\section{Exploitation of TDOA measurements}

The problem of passive emitter tracking can be considered in a network of senors as well. Various types of measurements can be obtained only with a network of sensors. TDOA measurements belong to this group. Several displaced, time-synchronized sensors measure the TOA of a signal transmitted from the emitter. The difference between two TOA measurements gives one TDOA measurement. In this chapter a network of two sensors building a sensor pair is regarded. They take measurements from an unknown emitter over time.

\subsection{Problem statement}

For a demonstration of the special features, the three-dimensional localization problem is not more enlightening than the two-dimensional one. Therefore, for easy understanding and presenting, the further text is restricted to the special case, where the trajectories of the sensors and the emitter lie in a plane.

Let $\mathbf{e}_{k}$ be the emitter state at time $t_{k}$ :

$$
\mathbf{e}_{k}=\left(\mathbf{x}_{k}^{T}, \dot{\mathbf{x}}_{k}^{T}\right)^{T}
$$


where $\mathbf{x}_{k}=\left(x_{k}, y_{k}\right)^{T} \in \mathbb{R}^{2}$ denotes the position and $\dot{\mathbf{x}}_{k}=\left(\dot{x}_{k}, \dot{y}_{k}\right)^{T} \in \mathbb{R}^{2}$ the velocity. Two sensors with the state vectors

$$
\mathbf{s}_{k}^{(i)}=\left(\mathbf{x}_{k}^{(i)^{T}}, \dot{\mathbf{x}}_{k}^{(i)^{T}}\right)^{T}, \quad i=1,2,
$$

observe the emitter and receive the emitted signal. The sensors have a navigation system to know their own position and speed. Therefore their state vectors are known at every time.

To simplify, the emitter is assumed to be stationary, i.e. $\dot{\mathbf{x}}_{k}=0$, while the sensors move along their trajectories with a constant speed.

The speed of propagation is the speed of light $c$, the TOA measurement can be expressed by:

$$
t_{0}+\frac{1}{c}\left\|\mathbf{x}_{k}-\mathbf{x}_{k}^{(i)}\right\|
$$

where $\|\cdot\|$ denotes the vector norm. $t_{0}$ is the emission time of the signal and $\left\|\mathbf{r}_{k}^{(i)}\right\|=$ $\left\|\mathbf{x}_{k}-\mathbf{x}_{k}^{(i)}\right\|$ is the range between emitter and sensor $i, i=1,2$, at time $t_{k}$, where $\mathbf{r}_{k}^{(i)}$ denotes the emitter position relative to sensor $i$.

The TOA measurement consists of the unknown time of emission $t_{0}$ and the time the signal needs for propagating the relative vector between the emitter and sensor $i$. Calculating the difference between the TOA measurements eliminates the unknown time $t_{0}$ and yields the TDOA measurement at time $t_{k}$ :

$$
h_{k}^{t}=\frac{1}{c}\left(\left\|\mathbf{x}_{k}-\mathbf{x}_{k}^{(1)}\right\|-\left\|\mathbf{x}_{k}-\mathbf{x}_{k}^{(2)}\right\|\right) .
$$

The measurement in the range domain is obtained by multiplication with the speed of the light $c$ :

$$
h_{k}^{\mathrm{r}}=\left\|\mathbf{x}_{k}-\mathbf{x}_{k}^{(1)}\right\|-\left\|\mathbf{x}_{k}-\mathbf{x}_{k}^{(2)}\right\| .
$$

The measurement equation is a function of the unknown emitter position $\mathbf{x}_{k}$, the emitter speed is not important. Furthermore, the positions of the sensors which are changing over time are parameters of the measurement equation, the sensor speed is irrelevant. The two-dimensional vector of position $\mathbf{x}_{k}$ of the emitter is to be estimated. The emitter is stationary, its position is independent of the time and it holds for all time step $t_{k}$ :

$$
\mathbf{x}_{k}=\mathbf{x}_{0} .
$$

A typical TDOA situation is illustrated in Figure 2. The two sensors move at the edge of the observation area in an easterly direction indicated by the arrows. They observe a stationary emitter. A single accurate, i.e. noise-free, TDOA measurement defines a hyperbola as possible emitter location. In Figure 2, the red curve shows the branch of the hyperbolae on which the emitter must be placed.

The combination of two measurements of the stationary emitter taken over time leads to an ambiguity of the emitter position. The two detection results are the true position of the emitter and the position mirrored along the connecting line between the sensors. This ambiguity can be resolved in various ways, e.g. by a maneuver of the sensors, the addition of a third sensor, or an additional bearing measurement. Alternatively, sensors which are sensitive only in the hemisphere can be used, and thus able to observe only this half-space. Here the sensors are positioned at the edge of the observation area, e.g. on a coast for the observation of a ground emitter or on the edge of a hostile territory. 


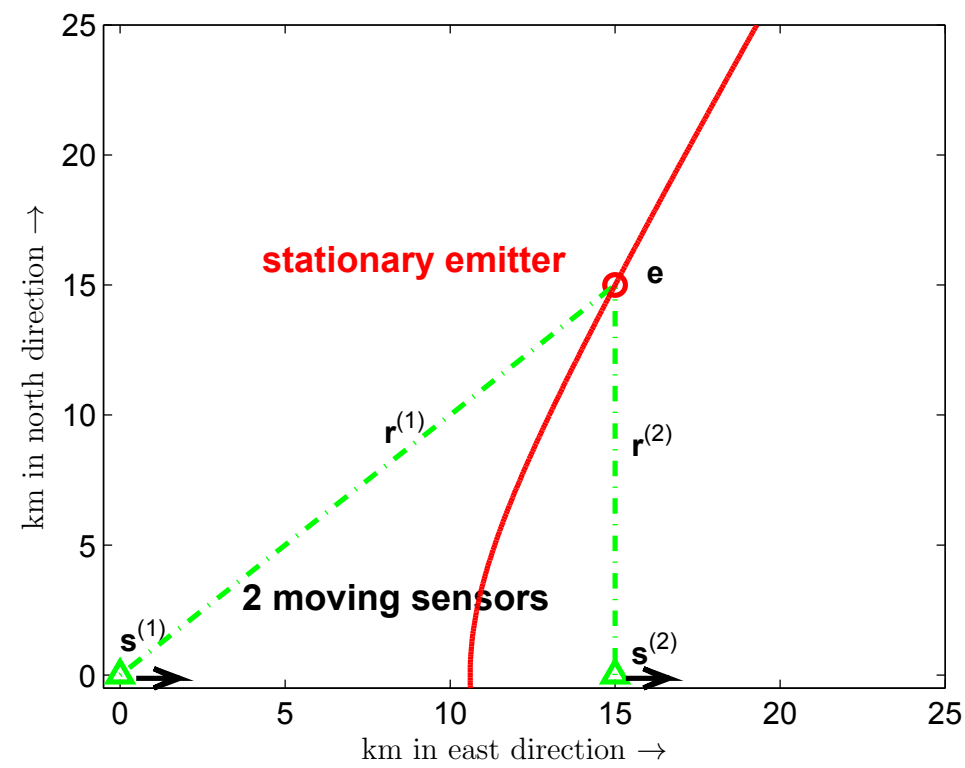

Fig. 2. TDOA scenario

The measurement process is modeled by adding white Gaussian noise to the measurement function. We get the measurement equation in the range domain at time $t_{k}$ :

$$
z_{k}^{\mathrm{r}}=h_{k}^{\mathrm{r}}+u_{k}^{\mathrm{r}} \quad u_{k}^{\mathrm{r}} \sim \mathcal{N}\left(0, \sigma_{r}^{2}\right)
$$

where $\sigma_{r}$ denotes the standard deviation of the measurement error in the range domain. The measurement noise $u_{k}^{\mathrm{r}}$ is i.i.d., the measurement error is independent from time to time, i.e. mutually independent, and identically distributed.

\subsection{Quantitative analysis}

Two different emitter tracking scenarios are considered to compare the performance of four different estimation algorithms which solve the nonlinear emitter localization problem, the results have already been published in (Kaune, 2009). The results presented here are based on 100 measurements averaged over 1000 independent Monte Carlo simulations with a measurement interval of two seconds. The measurement standard deviation in the range domain $\sigma_{r}$ is assumed to be $200 \mathrm{~m}$. This corresponds to a measurement standard deviation in the time domain $\sigma_{t}$ of about $0.67 \mu \mathrm{s}$.

In the first scenario, sensors, separated by a distance of $20 \mathrm{~km}$, fly one after the other in east direction at a constant speed of $100 \mathrm{~m} / \mathrm{s}$. The second scenario analyzes a parallel flight of the sensors. Sensors at $(1,1) \mathrm{km}$ and $(16,1) \mathrm{km}$ fly side by side in parallel at a constant speed of $100 \mathrm{~m} / \mathrm{s}$ in north direction. 


\subsubsection{CRLB investigation}

The CRLB for the TDOA scenario at time $t_{k}$ with the measurements $z_{i}$ and the time-dependent measurement functions $h\left(\mathbf{x}_{i}\right), i=1, \ldots, k$, can be computed as:

$$
\mathbf{J}_{k}=\frac{1}{\sigma_{r}^{2}} \sum_{i=1}^{k}\left(\frac{\partial h\left(\mathbf{x}_{i}\right)}{\partial \mathbf{x}_{k}}\right)^{T} \frac{\partial h\left(\mathbf{x}_{i}\right)}{\partial \mathbf{x}_{k}}
$$

with entries of the Jacobian at time $t_{i}$ :

$$
\frac{\partial h\left(\mathbf{x}_{i}\right)}{\partial x_{i}}=\frac{x_{i}-x_{i}^{(1)}}{\left\|\mathbf{r}_{i}^{(1)}\right\|}-\frac{x_{i}-x_{i}^{(2)}}{\left\|\mathbf{r}_{i}^{(2)}\right\|} \text { and } \frac{\partial h\left(\mathbf{x}_{i}\right)}{\partial y_{i}}=\frac{y_{i}-y_{i}^{(1)}}{\left\|\mathbf{r}_{i}^{(1)}\right\|}-\frac{y_{i}-y_{i}^{(2)}}{\left\|\mathbf{r}_{i}^{(2)}\right\|} .
$$

This shows that the CRLB depends only on the relative position of the sensors and the emitter, the measurement accuracy and the number of measurements.

The FIM $\mathbf{J}_{1}$ at time $t_{1}$ will usually be singular since we cannot estimate the full position vector $\mathbf{x}$ from a single TDOA measurement without additional assumptions, see (Van Trees, 1968). In the present case these assumptions concern the area in which the emitter is supposed to be. These assumptions about the prior distribution on $\mathbf{x}$ are added to the FIM at time $t_{1}$.

For visualization, the estimation accuracy is given as the square root of the trace of the $2 \times 2$ CRLB matrix.

Figure 3 shows a plot of the CRLB in the plane for the two investigated scenarios without taking into account of prior information. The initial sensor positions are marked with green triangles, and the red circle designates the position of the emitter. For a grid of possible emitter
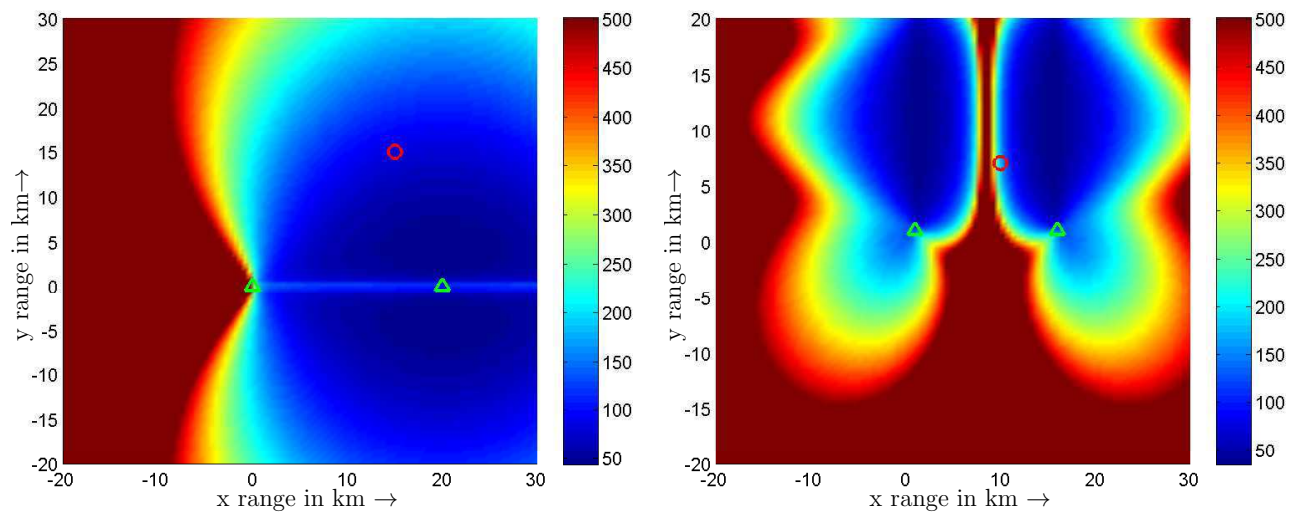

Fig. 3. CRLB in the plane, values cut off at $500 \mathrm{~m}$ : (a) scenario 1 (b) scenario 2, colorbar in $\mathrm{m}$.

positions in the plane the Fisher information $\mathbf{J}_{100}$ after 100 measurements is computed by Equation (6). The associated CRLB $\mathbf{J}_{100}^{-1}$ is calculated and the square root of the trace is shown. Values larger than $500 \mathrm{~m}$ have been cut off for better visualization. The color bar shows the localization accuracy in $\mathrm{m}$. The localization accuracy can be read from the figure for any emitter location in the plane.

In the first scenario, the emitter lies exactly in the area of optimal approach to the target. 
In the second scenario, it is near the region of divergence which indicates poor localization performance.

\subsubsection{Results}

For comparison of the estimation methods, the Root Mean Square Error (RMSE), the squared distance of the estimate to the true target location $\mathbf{x}_{k}$ is used in Monte Carlo simulations. They are averaged over $N$, the number of Monte Carlo runs. Let $\hat{\mathbf{x}}_{k}^{(i)}$ be the estimate of the $i$ th run at time $t_{k}$. Than, the RMSE at time $t_{k}$ is computed as:

$$
\operatorname{RMSE}_{k}=\sqrt{\frac{1}{N} \sum_{i=1}^{N}\left(\mathbf{x}_{k}-\hat{\mathbf{x}}_{k}^{(i)}\right)^{T}\left(\mathbf{x}_{k}-\hat{\mathbf{x}}_{k}^{(i)}\right)} .
$$

Four estimation algorithms which solve the nonlinear emitter localization problem are investigated and compared.

- The Maximum Likelihood Estimate (MLE) is that value of $\mathbf{x}_{k}$ which maximizes the likelihood function (30). Since there is no closed-form ML solution for $\mathbf{x}_{k}$, a numerical iterative search algorithm is needed to find the minimum of the quadratic form, see equation (42). In our case, the simplex method due to Nelder and Mead is used. It is initialized with a central point from the observation area in scenario 1, in the second scenario the initialization point is chosen at a distance of up to about $5 \mathrm{~km}$ from the true target position. Being a batch algorithm, the MLE evaluates, at each update, the complete measurement dataset. It attains the CRLB when properly initialized. One disadvantage of the ML estimator is the higher computational effort in comparison to the Kalman filters, as can be seen in Table 1. Table 1 shows the computational efforts of the different estimation algorithms for a Monte Carlo simulation with 1000 runs for the first scenario. One advantage of the MLE is the superior performance in comparison to the Kalman filters.

- The Extended Kalman filter (EKF) approximates the nonlinear measurement equation by its first-order Taylor series expansion:

$$
\widehat{\mathbf{H}}_{k}=\frac{\left(\mathbf{x}_{k}-\mathbf{x}_{k}^{(1)}\right)^{T}}{\left\|\mathbf{x}_{k}-\mathbf{x}_{k}^{(1)}\right\|}-\frac{\left(\mathbf{x}_{k}-\mathbf{x}_{k}^{(2)}\right)^{T}}{\left\|\mathbf{x}_{k}-\mathbf{x}_{k}^{(2)}\right\|} .
$$

Then, the Kalman filter equations are applied. The EKF is highly sensitive to the initialization and works only if the initial value is near the true target position. The EKF may not reach the CRLB even in the case of a good initialization. Initial values are chosen from a parametric approach similar to the approach described in (Mušicki \& Koch, 2008): the first measurement is used for initialization. It defines a hyperbola as possible emitter locations from which several points are taken. These points initialize a ML estimate which evaluates a sequence of first measurements. The best result is the initial value of the EKF and the UKF. The computational efforts shown in Table 1 include this phase of initialization.

- The Unscented Kalman filter (UKF) (see (Julier \& Uhlmann, 2004)) uses the Gaussian representation of the posterior density via a set of deterministically chosen sample points. These sample points are propagated through the Unscented Transform (UT). 
Since the nonlinearity is in the measurement equation, the UT is applied in the update step. Then the KF equations are carried out.

The initialization is the same as in the EKF. Poor initialization values result in divergent tracks like in the EKF case.

\begin{tabular}{|c|c|c|c|}
\hline \multicolumn{4}{|c|}{ Time in sec } \\
\hline EKF & UKF & MLE & GS \\
\hline 49 & 80 & 939 & 90 \\
\hline
\end{tabular}

Table 1. Comparison of computational effort

- The static Gaussian Mixture (GM) filter overcomes the initialization difficulties of the Kalman filter like EKF and UKF. It approximates the posterior density by a Gaussian Mixture (GM)((Tam et al., 1999)), a weighted sum of Gaussian density functions. The computational effort of finding a good initialization point is omitted here. The first measurement is converted into a Gaussian sum. The algorithmic procedure for computation of weights $w_{g}$, means $\mathbf{x}_{g}$ and covariances $\mathbf{P}_{g}$ is the same as in (Mušicki \& Koch, 2008). The mapping of the TDOA measurement into the Cartesian state space consists of several steps:

- present the $\pm \sigma_{r}$ hyperbolae in the state space,

- choose the same number of points on each hyperbolae,

- inscribe an ellipse in the quadrangle of two points on the $+\sigma_{r}$ and two points on the $-\sigma_{r}$ hyperbola,

- the center of the ellipse is the mean, the ellipse the covariance and the square root of the determinant the weight of the Gaussian summand.

An EKF is started for each mean and covariance, the weights are updated with the posterior probability. The final mean is computed as weighted sum of the individual EKF means: $\overline{\mathbf{x}}=\sum_{g=1}^{n} w_{g} \mathbf{x}_{g}$, where $n$ is the number of Gaussian terms.

The performance of these four estimation algorithms is investigated in two different tracking scenarios. In the first scenario, the emitter at $(15,15) \mathrm{km}$ lies in a well-locatable region. MLE shows good performance. The results of EKF and UKF are shown in Figure 4. They perform well and the NEES, see appendix A.2, lies in the 95\% interval $[1.878,2.126]$ for both filters, as can be seen from Figure 4 (b). For this scenario the static GM filter shows no improvement compared to a single EKF or UKF.

Scenario 2 analyzes a parallel flight of the sensors. The CRLB for the emitter position in $(10,7)$ $\mathrm{km}$ indicates poor estimation accuracy. EKF and UKF have heavy initialization problems, both have a high number of diverging tracks. Also the MLE suffers from difficulties of divergence. The initialization with a GM results in 9 simultaneously updated EKFs. The sampling from the GM approximation of the first measurement is presented in Figure 5 (a). The black solid lines are the $\pm \sigma_{r}$ hyperbolae. The sampling points are displayed in blue. They give an intelligent approximation of the first measurement. In Figure 5 (b) the RMSE of the GM filter and the MLE are plotted in comparison to the CRLB. In this scenario the GM filter, the bank of 9 EKFs, shows good performance. After an initial phase, it nears asymptotically the CRLB. The results of a single KF are unusable, they are higher than $10^{5} \mathrm{~m}$ and for better visibility 

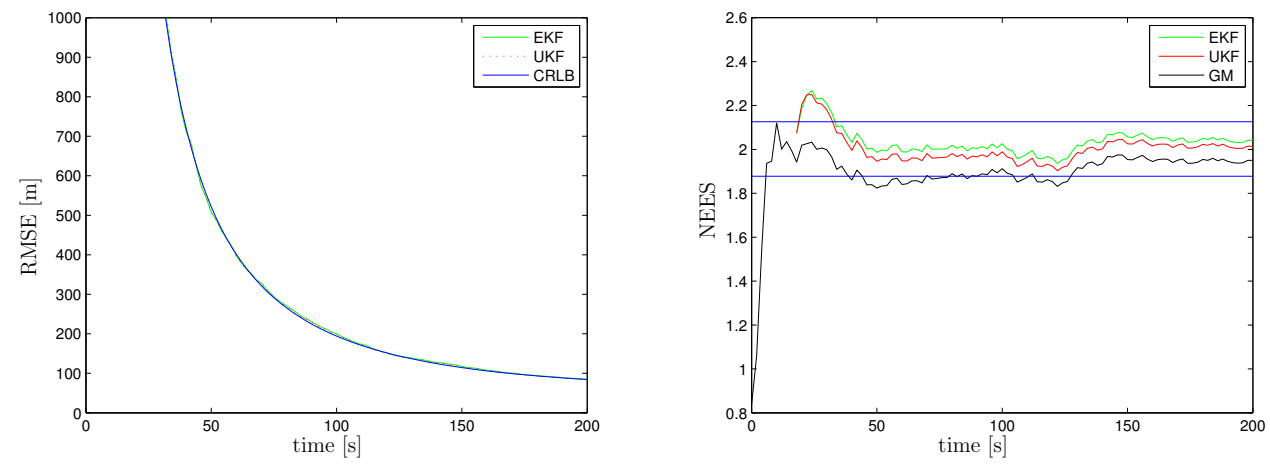

Fig. 4. (a) RMSE for EKF and UKF and (b) NEES for scenario 1

not presented. The MLE is initialized as described above and produces good results near the CRLB. Its performance is better than the performance of the GM filter. The CRLB are shown with initial assumptions.
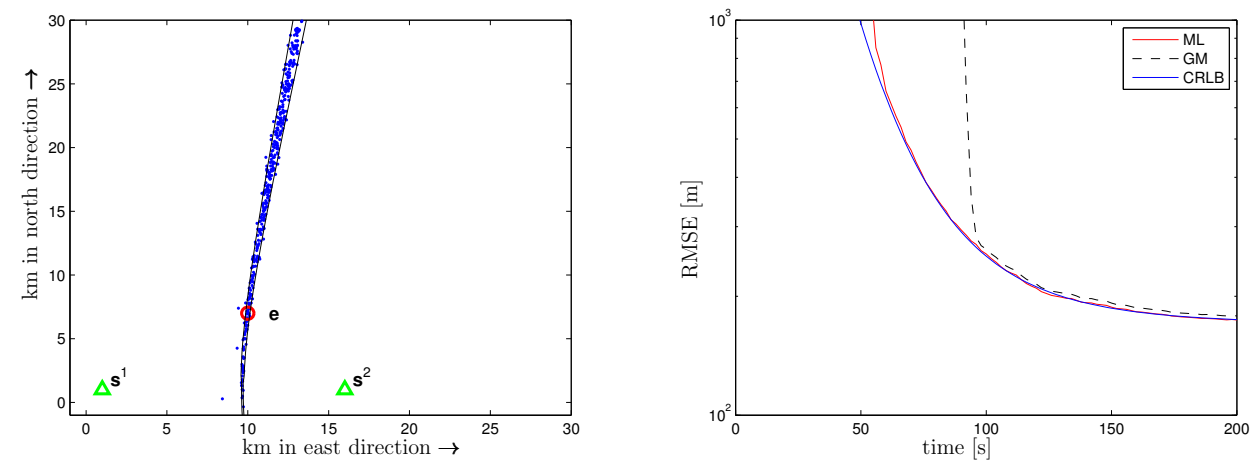

Fig. 5. (a) Sampling from the GM approximation and (b) RMSE for scenario 2

\section{Combination of TDOA and AOA measurements}

The combination of various types of measurements may lead to a gain in estimation accuracy. Particularly in the case of a moving emitter, it is advantageous to fuse different kinds of measurements. One possibility is that one sensor of the sensor pair is additionally able to take the bearing measurements.

\subsection{Problem statement}

Let $\mathbf{s}^{(1)}$ be the location of the sensor, which takes the bearing measurements. The additional azimuth measurement function at time $t_{k}$ is: 


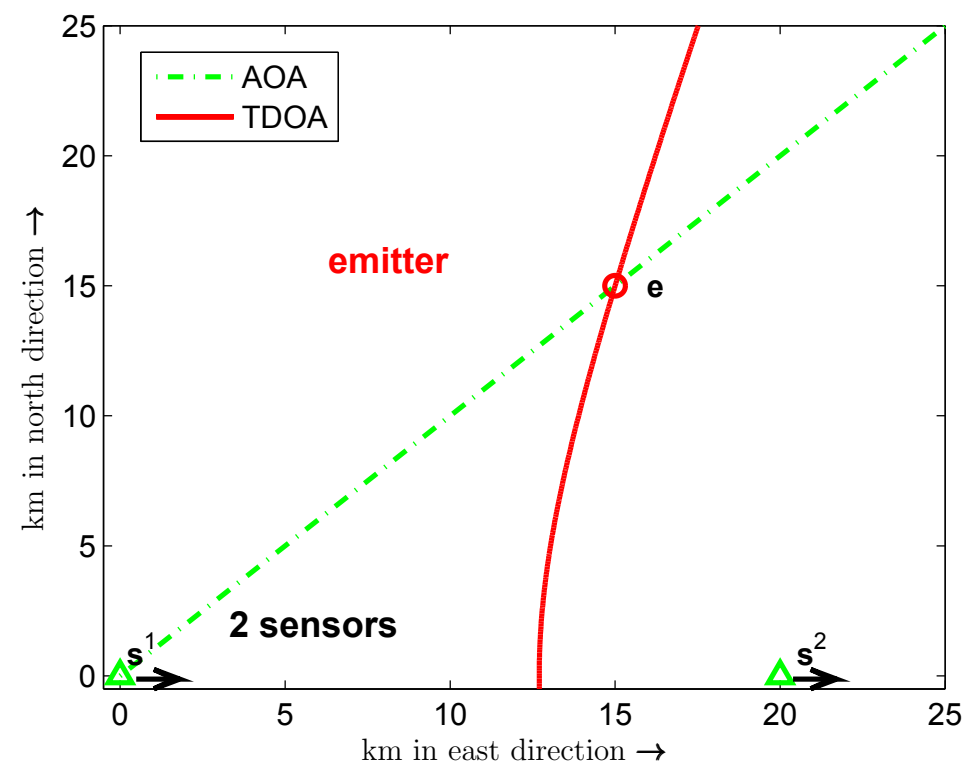

Fig. 6. Combination of one TDOA and one azimuth measurement

$$
h_{k}^{\alpha}=\arctan \left(\frac{x_{k}-x_{k}^{(1)}}{y_{k}-y_{k}^{(1)}}\right)
$$

Addition of white noise yields:

$$
z_{k}^{\alpha}=h_{k}^{\alpha}+u_{k}^{\alpha}, \quad u_{k}^{\alpha} \sim \mathcal{N}\left(0, \sigma_{\alpha}^{2}\right),
$$

where $\sigma_{\alpha}$ is the standard deviation of the AOA measurement.

Figure 6 shows the measurement situation after taking a pair of one azimuth and one TDOA measurement. At each time step, two nonlinear measurements are taken, which must be processed with nonlinear estimation algorithms.

\subsection{Quantitative analysis}

A moving emitter with one maneuver is considered to compare the performance of an estimator using single azimuth measurements and an estimator using the fused measurement set of azimuth and TDOA measurements. At the maneuvering time the emitter changes the flight direction and its velocity. The observer which takes the azimuth measurements flies at a constant speed of $50 \mathrm{~m} / \mathrm{s}$ on a circular trajectory for observability reasons, see Figure 7 . This sensor takes every 2 nd second azimuth measurements from the maneuvering emitter. TDOA measurements are gained from the network of the moving sensor and a stationary observer which lies in the observation space. TDOA measurements are also taken every 2 nd second. 
Thus, at each time step a pair of one azimuth and one TDOA measurement can be processed. The azimuth measurement standard deviation is assumed to be 1 degree and the TDOA measurement standard deviation is assumed to be $200 \mathrm{~m}$ in the range domain.

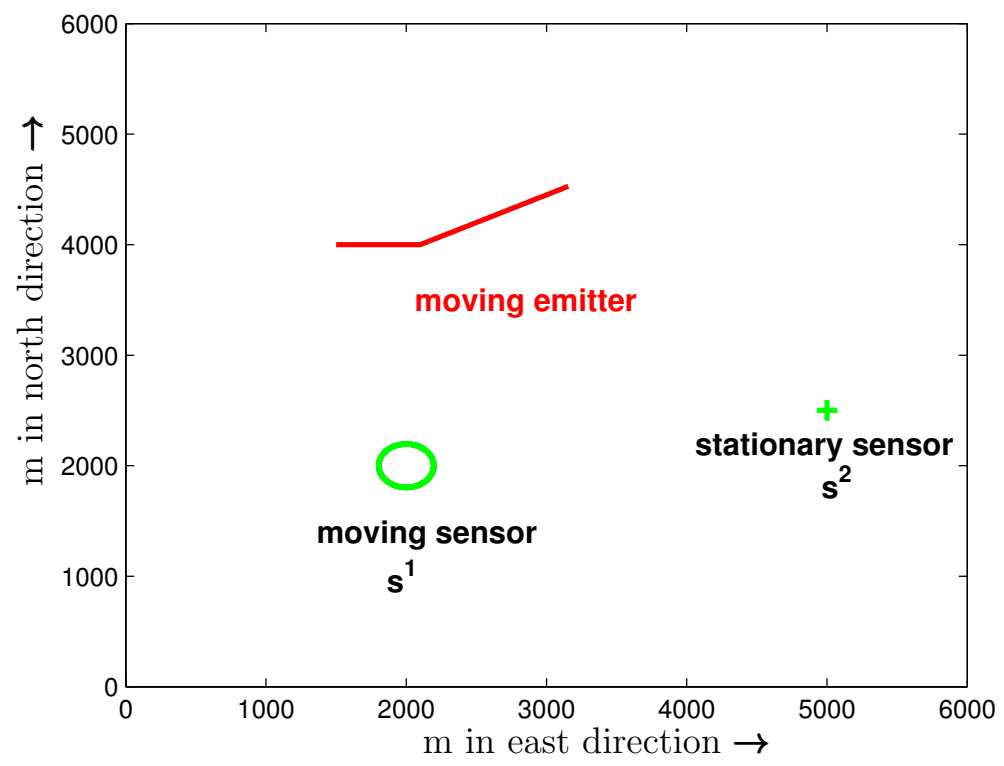

Fig. 7. Measurement situation

\subsubsection{CRLB investigation}

The CRLB of the combination of TDOA and AOA measurements is calculated over the fused Fisher information of the single Fisher informations. The Fisher information at time $t_{k}$ is the sum of the FIMs based on the TDOA and the AOA measurements:

$$
\mathbf{J}_{k}=\frac{1}{\sigma_{r}^{2}} \sum_{i=1}^{k}\left(\frac{\partial h^{r}\left(\mathbf{e}_{i}\right)}{\partial \mathbf{e}_{k}}\right)^{T} \frac{\partial h^{r}\left(\mathbf{e}_{i}\right)}{\partial \mathbf{e}_{k}}+\frac{1}{\sigma_{\alpha}^{2}} \sum_{i=1}^{k}\left(\frac{\partial h^{\alpha}\left(\mathbf{e}_{i}\right)}{\partial \mathbf{e}_{k}}\right)^{T} \frac{\partial h^{\alpha}\left(\mathbf{e}_{i}\right)}{\partial \mathbf{e}_{k}},
$$

with entries of the Jacobian of the AOA measurement equation:

$$
\begin{aligned}
& \frac{\partial h^{\alpha}\left(\mathbf{e}_{i}\right)}{\partial x_{i}}=\frac{y_{i}-y_{i}^{(1)}}{\left\|\mathbf{r}_{i}^{(1)}\right\|^{2}} \\
& \frac{\partial h^{\alpha}\left(\mathbf{e}_{i}\right)}{\partial y_{i}}=-\frac{x_{i}-x_{i}^{(1)}}{\left\|\mathbf{r}_{i}^{(1)}\right\|^{2}} \\
& \frac{\partial h^{\alpha}\left(\mathbf{e}_{i}\right)}{\partial \dot{x}_{i}}=\frac{\partial h^{\alpha}\left(\mathbf{e}_{i}\right)}{\partial \dot{y}_{i}}=0 .
\end{aligned}
$$

Therefore, the localization accuracy depends on the sensor-emitter geometry, the standard deviation of the TDOA and the azimuth measurements and the number of measurements. 


\subsubsection{Results}

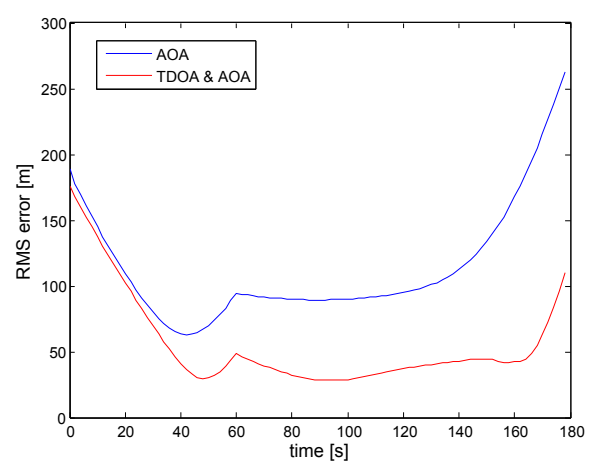

(a) MLE

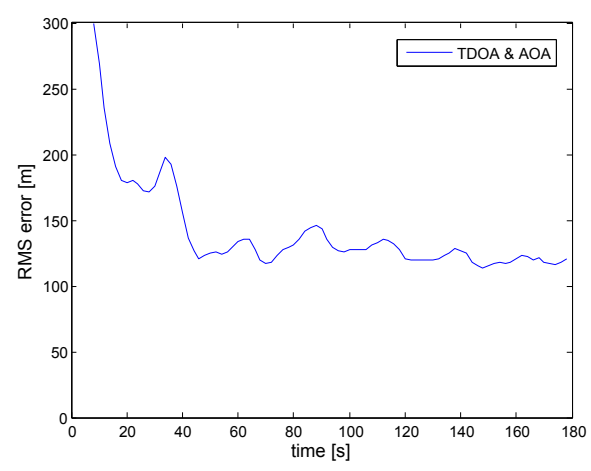

(b) UT

Fig. 8. Comparison of AOA and a combination of AOA and TDOA

Three estimation algorithms are compared:

- MLE based on azimuth-only measurements: It works with knowledge of the emitter dynamic: the state of the target is modeled using the dynamic with one maneuver and of a constant velocity before and after the maneuvering time. The 7-dimensional emitter state is to be estimated, including the maneuvering time and the two speed vectors of the two segments of the emitter trajectory. The modeling of the emitter dynamic and the algorithms for the MLE are implemented like in (Oispuu \& Hörst, 2010), where piecewise curvilinearly moving targets are considered. The processing of the measurements is done after taking the complete measurement dataset in retrospect. The 7-dimensional emitter state can be computed for every time step or alternatively for a single reference time step.

- MLE based on the combination of azimuth and TDOA measurements. The algorithm is the same algorithm as for the AOA only case. The TDOA measurements are basis of the optimization, too.

- A filter which uses the combined measurement set of azimuth and TDOA measurements: it transforms at each time step the measurement pair of azimuth and TDOA measurement $\left\{z^{\alpha}, z^{t}\right\}$ into the Cartesian state space. At each time step, using the UT an estimation of the emitter state in the Cartesian state space and an associated covariance are obtained. Emitter tracking is started with the first measurement pair and performed in parallel to gaining the measurements.

The UT consists of two steps: 
- Computation of the distance from sensor $\mathbf{s}^{(1)}$ to the emitter:

$$
\left\|\mathbf{r}^{(1)}\right\|=\frac{\left\|\mathbf{x}^{(1)}-\mathbf{x}^{(2)}\right\|^{2}-z^{t^{2}}}{2\left[\left(\mathbf{x}^{(2)}-\mathbf{x}^{(1)}\right)^{T}\left(\begin{array}{l}
\sin \left(z^{\alpha}\right) \\
\cos \left(z^{\alpha}\right)
\end{array}\right)-z^{t}\right]}
$$

- Calculation of the emitter location:

$$
\hat{\mathbf{x}}=\mathbf{x}^{(1)}+\left\|\mathbf{r}^{(1)}\right\|\left(\begin{array}{c}
\cos \left(z^{\alpha}\right) \\
\sin \left(z^{\alpha}\right)
\end{array}\right) ;
$$

The measurement pair and its associated measurement covariance $\mathbf{R}=\operatorname{diag}\left[\sigma_{\alpha}^{2}, \sigma_{r}^{2}\right]$, where diag[] means the diagonal matrix, is processed using the UT. I.e., several sigma points in the two-dimensional measurement space are selected and transformed. We obtain an estimation of the emitter state in the Cartesian state space and an associated covariance. A linear Kalman filter is started with the position estimate and the associated covariance. The update is performed in the Cartesian state space by transforming the incoming measurement pair using the unscented transform. This filter uses as model for the emitter dynamic the model for a inertially moving target. This model does not describe correctly the emitter dynamic but addition of white Gaussian process noise can correct the error of the model.

In Figure 8 the results based on 1000 Monte Carlo runs are presented. Figure 8 (a) shows the comparison between the MLE only based on azimuth measurements and based on a combination of azimuth and TDOA measurements. The MLE delivers for each Monte Carlo run one 7-dimensional estimate of the emitter state from which the resulting emitter trajectory is computed. The RMS error to the true emitter trajectory is shown. Using the combined measurement set, the performance is significant better than the AOA only results. Figure 8 (b) visualizes the results of the linear KF using the UT. At each time step, an estimate of the emitter state is computed. In spite of an insufficient dynamic model, the emitter state is estimated quite fair in the beginning. But due to the incorrect dynamic model, the localization accuracy in the end is about $120 \mathrm{~m}$. The MLE based on the combined measurement set shows better performance than the filter using the UT.

\section{Combination of TDOA and FDOA measurements}

A combination of TDOA and FDOA measurements increases the performance compared to single TDOA measurements (see (Mušicki et al., 2010)). A minimum of two sensors is needed to gain FDOA measurements at one time step. The omnidirectional antennas which measure the TOA can measure the frequency of the received signal as well. Frequency measurements depend on the relative motion between the emitter and the sensors. The radial component of the relative speed vector determines the frequency shift which is necessary to obtain nonzero FDOA values.

\subsection{Problem statement}

The FDOA measurement function depends not only on the emitter position but also on its speed and course, for easy understanding the subscript $k$ for the time step is omitted in this 


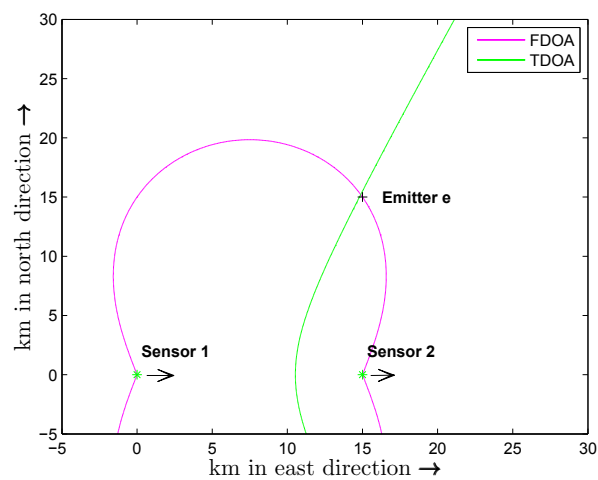

(a) tail flight

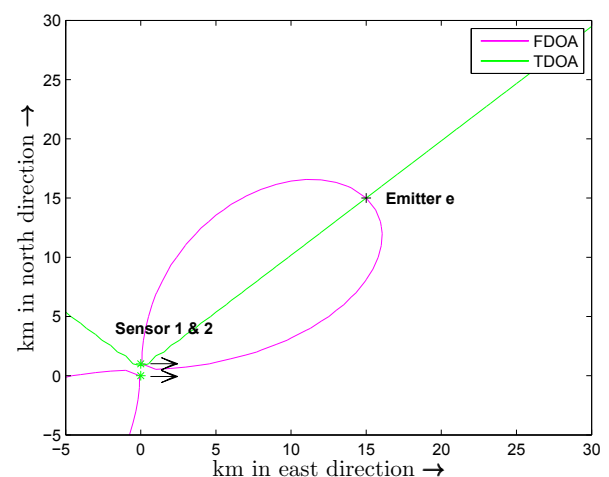

(b) parallel flight

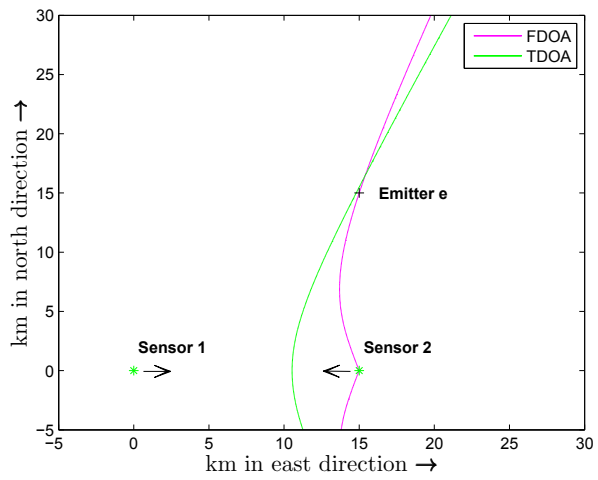

(c) flight head on

Fig. 9. Combination of TDOA and FDOA measurements in three different scenarios

section if it is clear from the context:

$$
h^{f f}=\frac{f_{0}}{c}\left(\left(\dot{\mathbf{x}}^{(1)}-\dot{\mathbf{x}}\right)^{T} \frac{\mathbf{r}^{(1)}}{\left\|\mathbf{r}^{(1)}\right\|}-\left(\dot{\mathbf{x}}^{(2)}-\dot{\mathbf{x}}\right)^{T} \frac{\mathbf{r}^{(2)}}{\left\|\mathbf{r}^{(2)}\right\|}\right),
$$

where $f_{0}$ is carrier frequency of the signal. Multiplication with $\frac{c}{f_{0}}$ yields the measurement equation in the velocity domain:

$$
h^{f}=\left(\dot{\mathbf{x}}^{(1)}-\dot{\mathbf{x}}\right)^{T} \frac{\mathbf{r}^{(1)}}{\left\|\mathbf{r}^{(1)}\right\|}-\left(\dot{\mathbf{x}}^{(2)}-\dot{\mathbf{x}}\right)^{T} \frac{\mathbf{r}^{(2)}}{\left\|\mathbf{r}^{(2)}\right\|} .
$$

Under the assumption of uncorrelated measurement noise from time step to time step and from the TDOA measurements, we obtain the FDOA measurement equation in the velocity domain:

$$
z^{f}=h^{f}+u_{f}, \quad u_{f} \sim \mathcal{N}\left(0, \sigma_{f}^{2}\right),
$$


where $\sigma_{f}$ is the standard deviation of the FDOA measurement. The associated TDOA/ FDOA measurement pairs may be obtained by using the CAF ((Stein, 1981)). For each TDOA value the associated FDOA value can be calculated. Nonlinear estimation algorithms are needed to process the pair of TDOA and FDOA measurements and to estimate the emitter state.

Figure 9 shows the situation for different sensor headings after taking one pair of TDOA and FDOA measurements. The green curve, i.e. the branch of hyperbola, indicates the ambiguity after the TDOA measurement. The ambiguity after the FDOA measurement is plotted in magenta. The intersection of both curves presents a gain in information for the emitter location. This gain is very high if sensors move behind each other.

\subsection{Quantitative analysis}

In the following, a scenario with a moving emitter is investigated to compare the performance of two filters which exploit a combination of TDOA and FDOA and one filter based on single TDOA measurements. The presented filters are GM filters which approximate the required densities by a weighted sum of Gaussian densities.

\subsubsection{CRLB investigation}

The Fisher information at time $t_{k}$ is the sum of the Fisher information based on the TDOA and the FDOA measurements:

$$
\mathbf{J}_{k}=\frac{1}{\sigma_{r}^{2}} \sum_{i=1}^{k}\left(\frac{\partial h^{r}\left(\mathbf{e}_{i}\right)}{\partial \mathbf{e}_{k}}\right)^{T} \frac{\partial h^{r}\left(\mathbf{e}_{i}\right)}{\partial \mathbf{e}_{k}}+\frac{1}{\sigma_{f}^{2}} \sum_{i=1}^{k}\left(\frac{\partial h^{f}\left(\mathbf{e}_{i}\right)}{\partial \mathbf{e}_{k}}\right)^{T} \frac{\partial h^{f}\left(\mathbf{e}_{i}\right)}{\partial \mathbf{e}_{k}},
$$

with entries of the Jacobian of the FDOA measurement equation:

$$
\begin{aligned}
& \frac{\partial h^{f}(\mathbf{e})}{\partial x}=D_{x}^{(1)}-D_{x}^{(2)} \\
& \frac{\partial h^{f}(\mathbf{e})}{\partial y}=D_{y}^{(1)}-D_{y}^{(2)} \\
& \frac{\partial h^{f}(\mathbf{e})}{\partial \dot{x}}=\frac{x-x^{(2)}}{\left\|\mathbf{r}^{(2)}\right\|}-\frac{x-x^{(1)}}{\left\|\mathbf{r}^{(1)}\right\|} \\
& \frac{\partial h^{f}(\mathbf{e})}{\partial \dot{y}}=\frac{y-y^{(2)}}{\left\|\mathbf{r}^{(2)}\right\|}-\frac{y-y^{(1)}}{\left\|\mathbf{r}^{(1)}\right\|},
\end{aligned}
$$

with

$$
\begin{aligned}
& D_{x}^{(i)}=\frac{\left(\dot{x}^{(i)}-\dot{x}\right)-\frac{1}{\left\|\mathbf{r}^{(i)}\right\|^{2}}\left[\left(\dot{\mathbf{x}}^{(i)}-\dot{\mathbf{x}}\right)^{T} \mathbf{r}^{(i)}\right]\left(x-x^{(i)}\right)}{\left\|\mathbf{r}^{(i)}\right\|}, \quad i=1,2 \\
& D_{y}^{(i)}=\frac{\left(\dot{y}^{(i)}-\dot{y}\right)-\frac{1}{\left\|\mathbf{r}^{(i)}\right\|^{2}}\left[\left(\dot{\mathbf{x}}^{(i)}-\dot{\mathbf{x}}\right)^{T} \mathbf{r}^{(i)}\right]\left(y-y^{(i)}\right)}{\left\|\mathbf{r}^{(i)}\right\|}, \quad i=1,2 .
\end{aligned}
$$

The CRLB depends not only on the sensors-emitter geometry and the measurement standard deviations but also on the velocity. A nonzero radial component of the relative velocity vector is needed to obtain nonzero FDOA values.

The CRLB for one time scan, a pair of one TDOA and one FDOA measurement, is plotted 
in Figure 10. Assumed is a standard deviation of TDOA of $200 \mathrm{~m}(0.67 \mu \mathrm{s})$ and a standard deviation of FDOA of $4 \mathrm{~m} / \mathrm{s}$, this corresponds to a standard deviation in the frequency domain of $40 \mathrm{~Hz}$, assuming a carrier frequency of about $3 \mathrm{GHz}$. The color bar shows the values for the localization accuracy in $\mathrm{m}$. In these situations, the maximal gain in localization accuracy is obtained when the sensors fly one after the other. The results for the parallel flight can be improved if the distance of the sensors is increased.

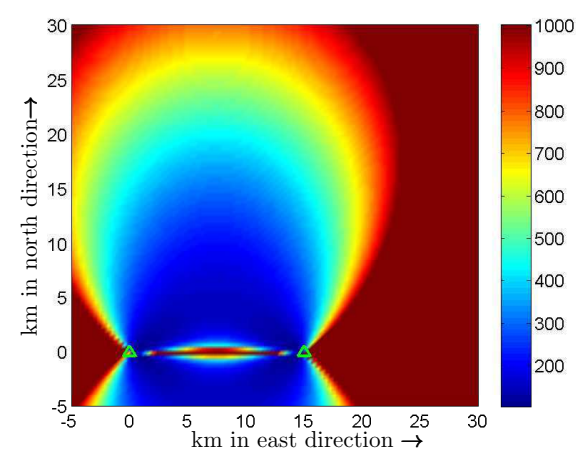

(a) tail flight

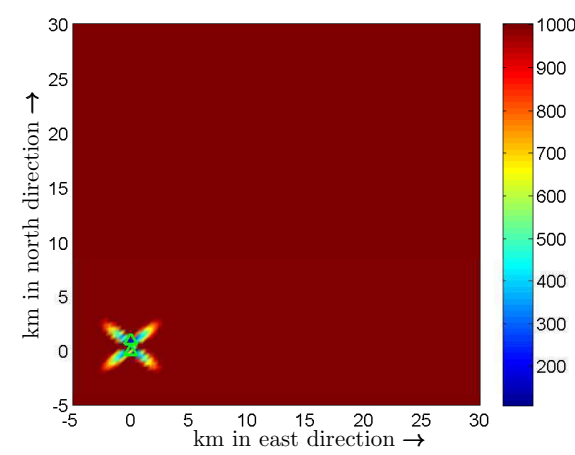

(b) parallel flight

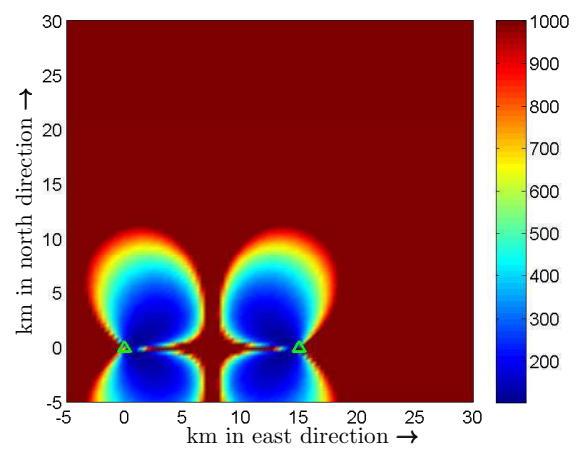

(c) flight head on

Fig. 10. CRLB for the combination of TDOA and FDOA for one time scan

\subsubsection{Results}

Both TDOA and FDOA measurement equations are nonlinear. Therefore nonlinear estimation algorithms are needed to process the combined measurement set. The performance of three different estimation algorithms is investigated in a scenario with a moving emitter.

The investigated scenario and the results are described in (Mušicki et al., 2010). The emitter is assumed to move at a constant speed in $x$-direction of $-10 \mathrm{~m} / \mathrm{s}$. Due to observability reasons, sensors perform maneuvers, they move with a constant speed, but not velocity, of $100 \mathrm{~m} / \mathrm{s}$. The results shown here are the product of a Monte Carlo simulation with 1000 runs with a 
sampling interval of two seconds. A total of $80 \mathrm{~s}$ is regarded, the maneuver is performed at $40 \mathrm{~s}$. The maximum emitter speed constraint is set to $V_{\max }=15 \mathrm{~m} / \mathrm{s}$. Measurement standard deviation $\sigma_{r}$ for TDOA is assumed to be $100 \mathrm{~m}$ in the range domain, and the standard deviation $\sigma_{f}$ for FDOA is assumed to be $10 \mathrm{~mm} / \mathrm{s}$ in the velocity domain.

The three investigated algorithms are:

- The GMM-ITS (Gaussian Mixture Measurement presentation-Integrated Track Splitting) filter using TDOA and FDOA measurements is a dynamic GM filter with integrated track management, see (Mušicki et al., 2010) (TFDOA in Figure 11). In (Mušicki et al., 2010) is demonstrated that the simultaneous processing of the measurement pair is equivalent to processing first the TDOA measurement and than the FDOA measurement. The filter is initialized with the GM approximation of the first TDOA measurement. One cycle of the filter consists of several steps:

- prior GM approximation of the updated density in the state space computed using previous measurements

- prediction in the state space for each component of the GM

- filtering with the incoming TDOA measurement:

(a) GM representation of the TDOA measurement,

(b) new components of the estimated state is obtained by updating each component of the predicted state space by each component of the TDOA GM,

(c) control of the number of new estimated state components (pruning and merging)

- filtering with the incoming FDOA measurement: each component of the state presentation is filtered with an EKF: updated density

- The GMM-ITS filter using single TDOA is a dynamic GM filter using only TDOA measurements. The processing is the same as in the GMM ITS filter, where the update process is only done with the TDOA measurements (TDOA in Figure 11)

- The static GM filter (fixed number of components) based on the combination of TDOA and FDOA measurements (static GM in Figure 11). The filter is initialized with the presentation of the TDOA measurement as a GM. The update is performed as EKF for the TDOA measurement as well as for the FDOA measurement, the filter based only on TDOA measurements is presented in 3.2.2.

Figure 11 presents the RMSE of the three described filters in comparison to the CRLB. The period after the sensor maneuvers, when the RMSE decreases, is zoomed in. In this scenario of a moving emitter, the filter based only on TDOA measurements shows poor performance. The combination of the various measurement types of TDOA and FDOA increases the estimation accuracy significantly. The static GM filter shows good performance with estimation errors of about $30 \mathrm{~m}$. The dynamic GM filter is nearly on the CRLB in the final phase with estimation errors of about $10 \mathrm{~m}$. This shows the significant gain in estimation accuracy combing different types of measurements. 

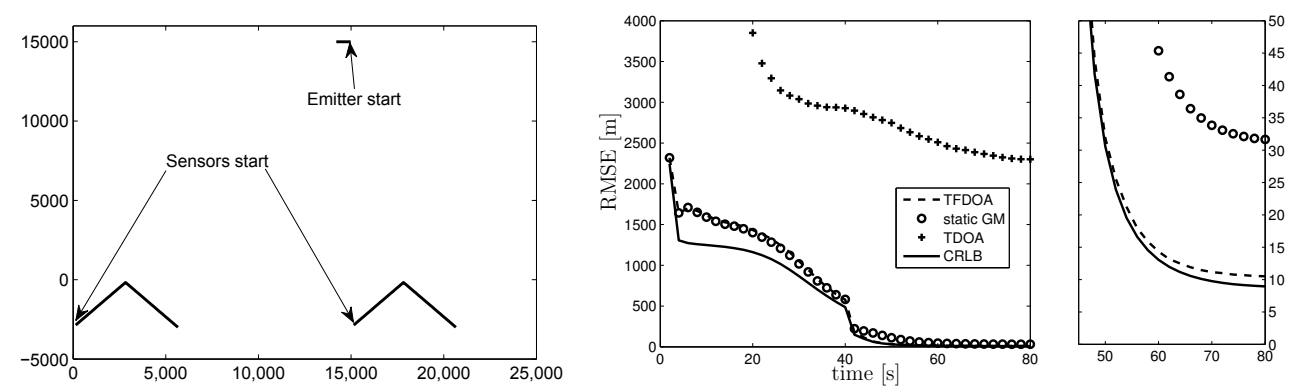

Fig. 11. (a) Scenario, (b) RMSE of the mobile emitter tracking (@[2010] IEEE) ${ }^{1}$

\section{Conclusions}

Passive emitter tracking in sensor networks is in general superior to emitter tracking using single sensors. Even a pair of sensors improves the performance strongly. The techniques of solving the underlying tracking problem are the same as in the single sensor case. The first step should be the investigation of the CRLB to know the optimal achievable estimation accuracy using the available measurement set. It reveals characteristic features of localization and gives an insight into the parametric dependencies of the passive emitter tracking problem under consideration. It shows that the estimation accuracy is often strongly dependent on the geometry. Secondly, a powerful estimation algorithm is needed to solve the localization problem. In passive emitter tracking, states and measurements are not linearly related. Therefore, only methods that appropriately deal with nonlinearities can be used. This chapter provides a review of different nonlinear estimation methods. Depending on the type of measurement and on different requirements in various scenarios, different estimation algorithms can be the methods of choice. E.g., to obtain good results near the CRLB the MLE is an appropriate method. Here, the computational effort is higher compared to alternatives such as Kalman filters. Tracking from the first measurement is possible using the UT in the TDOA/AOA case or using the GM filter or the GMM-ITS filter. They overcome the initialization difficulties of single Kalman Filters. The UT transform the measurement into the Cartesian state space and the GM filter and GMM-ITS filter approximate the first measurement by a Gaussian Mixture, a weighted sum of Gaussian densities. The first measurement is transformed into the Cartesian space and converted into a Gaussian sum. The tracking with the GM filter and GMM-ITS filter shows good performance and results near the CRLB.

For passive emitter tracking in sensor networks different measurement types can be gained by exploiting the signal coming from the target. Some of them can be taken by single sensors: e. g. bearing measurements. Others are only gained in the sensor network, a minimum of two sensors is needed. The combination of different measurements leads to a significant gain in estimation accuracy.

\section{References}

Bar-Shalom, Y., Li, X. R. \& Kirubarajan, T. (2001). Estimation with Applications to Tracking and Navigation: Theory Algorithms and Software, Wiley \& Sons.

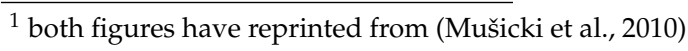


Becker, K. (1992). An Efficient Method of Passive Emitter Location, IEEE Trans. Aerosp. Electron. Syst. 28(4): 1091-1104.

Becker, K. (1993). Simple Linear Theory Approach to TMA Observability, IEEE Trans. Aerosp. Electron. Syst. 29, No. 2: 575-578.

Becker, K. (1996). A General Approach to TMA Observability from Angle and Frequency Measurements, IEEE Trans. Aerosp. Electron. Syst. 32, No. 1: 487-494.

Becker, K. (1999). Passive Localization of Frequency-Agile Radars from Angle and Frequency Measurements, IEEE Trans. Aerosp. Electron. Syst. 53, No. 4: 1129 - 1144.

Becker, K. (2001). Advanced Signal Processing Handbook, chapter 9: Target Motion Analysis (TMA), pp. 1-21.

Becker, K. (2005). Three-Dimensional Target Motion Analysis using Angle and Frequency Measurements, IEEE Trans. Aerosp. Electron. Syst. 41(1): 284-301.

Julier, S. J. \& Uhlmann, J. K. (2004). Unscented Filtering and Nonlinear Estimation, Proc. IEEE 92(3): 401-422.

K. C. Ho, L. Y. (2008). On the Use of a Calibration Emitter for Source Localization in the Presence of Sensor Position Uncertainty, IEEE Trans. on Signal Processing 56, No. 12: 5758 -5772 .

Kaune, R. (2009). Gaussian Mixture (GM) Passive Localization using Time Difference of Arrival (TDOA), Informatik 2009 - Workshop Sensor Data Fusion: Trends, Solutions, Applications.

Mušicki, D., Kaune, R. \& Koch, W. (2010). Mobile Emitter Geolocation and Tracking Using TDOA and FDOA Measurements, IEEE Trans. on Signal Processing 58, Issue 3, Part 2: $1863-1874$.

Mušicki, D. \& Koch, W. (2008). Geolocation using TDOA and FDOA measurements, Proc. 11th International Conference on Information Fusion, pp. 1-8.

Oispuu, M. \& Hörst, J. (2010). Azimuth-only Localization and Accuracy Study for Piecewise Curvilinearly Moving Targets, International Conference on Information Fusion.

Ristic, B., Arulampalam, S. \& Gordon, N. (2004). Beyond the Kalman Filter, Particle Filters for Tracking Applications, Artech House.

So, H. C., Chan, Y. T. \& Chan, F. K. W. (2008). Closed-Form Formulae for Time-Difference-ofArrival Estimation, IEEE Trans. on Signal Processing 56, No. 6: 2614- 2620.

Stein, S. (1981). Algorithms for Ambiguity Function Processing, IEEE Trans. Acoustic, Speech and Signal Processing 29(3): 588-599.

Tam, W. I., Plataniotis, K. N. \& Hatzinakos, D. (1999). An adaptive Gaussian sum algorithm for radar tracking, Elsevier Signal Processing 77: 85 - 104.

Van Trees, H. L. (1968). Detection, Estimation and Modulation Theory, Part I, New York: Wiley \& Sons.

\section{A. Appendix: Toolbox of methods}

\section{A.1 Cramér Rao investigation}

It is important to know the optimum achievable localization accuracy that can be attained with the measurements. This optimum estimation accuracy is given by the Cramér Rao lower bound (CRLB); it is a lower bound for an unbiased estimator and can be asymptotically achieved by unbiased estimators (Bar-Shalom et al., 2001; Van Trees, 1968). The investigation of the CRLB reveals characteristic features of the estimation problem under consideration. The CRLB can be used as a benchmark to asses the performance of the investigated estimation 
methods. The CRLB is calculated from the inverse of the Fisher Information Matrix (FIM) J. The CR inequality reads:

$$
\begin{gathered}
\mathbb{E}\left[\left(\hat{\mathbf{e}}_{k}-\mathbf{e}_{k}\right)\left(\hat{\mathbf{e}}_{k}-\mathbf{e}_{k}\right)^{T}\right] \geq \mathbf{J}_{k}^{-1}, \\
\mathbf{J}_{k}=\mathbb{E}\left[\nabla_{\mathbf{e}_{k}} \ln p\left(Z_{k} \mid \mathbf{e}_{k}\right)\left(\nabla_{\mathbf{e}_{k}} \ln p\left(Z_{k} \mid \mathbf{e}_{k}\right)\right)^{T}\right],
\end{gathered}
$$

where ê determines the estimate and $\mathbb{E}[\cdot]$ determines the expectation value.

The Fisher information $\mathbf{J}$ uses the Likelihood function, the conditional probability $p\left(Z_{k} \mid \mathbf{e}_{k}\right)$, for calculation:

$$
p\left(Z_{k} \mid \mathbf{e}_{k}\right)=\left(\frac{1}{\sqrt{\operatorname{det}(2 \pi \mathbf{R})}}\right) \exp \left(-\frac{1}{2} \sum_{i=1}^{k}\left(z_{i}-h\left(\mathbf{e}_{i}\right)\right)^{T} \mathbf{R}^{-1}\left(z_{i}-h\left(\mathbf{e}_{i}\right)\right)\right),
$$

where $Z_{k}=\left\{z_{1}, z_{2}, \ldots, z_{k}\right\}$ is the set of measurements up to time $t_{k}$. Under the assumption of non-correlation of the measurement noise from time to time the calculation of the CRLB is performed for the reference time $t_{k}$ with the measurement set $Z_{k}=\left\{z_{1}, \ldots, z_{k}\right\}$ and the time dependent measurement functions $\left\{h\left(\mathbf{e}_{1}\right), \ldots, h\left(\mathbf{e}_{k}\right)\right\}$. The computation results from the inverse of the Fisher information $\mathbf{J}_{k}$ at reference time $t_{k}$ :

$$
\mathbf{J}_{k}=\sum_{i=1}^{k}\left(\frac{\partial h\left(\mathbf{e}_{i}\right)}{\partial \mathbf{e}_{k}}\right)^{T} \mathbf{R}^{-1} \frac{\partial h\left(\mathbf{e}_{i}\right)}{\partial \mathbf{e}_{k}}
$$

where

$$
\frac{\partial h\left(\mathbf{e}_{i}\right)}{\partial \mathbf{e}_{k}}=\frac{\partial h\left(\mathbf{e}_{i}\right)}{\partial \mathbf{e}_{i}} \frac{\partial \mathbf{e}_{i}}{\partial \mathbf{e}_{k}} .
$$

For the stationary scenario the state vector $\mathbf{e}$ of the emitter is the same at each time step. That means,

$$
\frac{\partial h\left(\mathbf{e}_{i}\right)}{\partial \mathbf{e}_{i}}=\frac{\partial h\left(\mathbf{e}_{i}\right)}{\partial \mathbf{e}_{k}} \quad \forall i .
$$

For the mobile emitter case, we obtain, using the dynamic equation of the inertially target motion,

$$
\mathbf{e}_{k}=\mathbf{F}_{k \mid k-1} \mathbf{e}_{k-1}
$$

where $\mathbf{F}_{k \mid k-1}$ is the evolution matrix which relates the target state from time $t_{k}$ to time $t_{k-1}$, the FIM at reference time $t_{k}$

$$
\mathbf{J}_{k}=\sum_{i=1}^{k} \mathbf{F}_{k \mid i}^{-1 T}\left(\frac{\partial h\left(\mathbf{e}_{i}\right)}{\partial \mathbf{e}_{i}}\right)^{T} \mathbf{R}^{-1}\left(\frac{\partial h\left(\mathbf{e}_{i}\right)}{\partial \mathbf{e}_{i}}\right) \mathbf{F}_{k \mid i}^{-1} .
$$

At time $t_{1}$ the FIM $\mathbf{J}_{1}$ is usually singular and not invertible, because the state vector $\mathbf{e}_{k}$ cannot be estimated based on a single measurement without additional assumptions. Thus, we incorporate additional assumptions. These assumptions may concern the area in which the emitter is attended to be. This prior information about a prior distribution of $\mathbf{e}$ can be added to the FIM at time $t_{1}$ as artificial measurement:

$$
\mathbf{J}_{1}^{p r}=\mathbf{J}_{1}+\mathbf{J}_{p r}
$$

where $\mathbf{J}_{p r}$ is the prior Fisher information. Under the Gaussian assumption of $\mathbf{e}$ follows:

$$
\mathbf{J}_{p r}=\mathbf{P}_{p r}^{-1}
$$


where $\mathbf{P}_{p r}$ is the covariance of the prior distribution.

The prior information reduces the bound in the initial phase, but has little impact on later time steps.

\section{A.2 NEES}

Consistency is necessary for filter functionality, thus the normalized estimation error squared, the NEES is investigated, see (Bar-Shalom et al., 2001). A consistent estimator describes the size of the estimation error by its associated covariance matrix adequately. Filter consistency is necessary for the practical applicability of a filter.

The computation of the NEES requires the state estimate $\mathbf{e}_{k \mid k}$ at time $t_{k}$, its associated covariance matrix $\mathbf{P}_{k \mid k}$ and the true state $\mathbf{e}_{k}$.

Let $\tilde{\mathbf{e}}_{k \mid k}$ be the error of $\mathbf{e}_{k \mid k}: \tilde{\mathbf{e}}_{k \mid k}:=\mathbf{e}_{k}-\mathbf{e}_{k \mid k}$. The NEES is defined by this term:

$$
\epsilon_{k}=\tilde{\mathbf{e}}_{k \mid k}^{T} \mathbf{P}_{k \mid k}^{-1} \tilde{\mathbf{e}}_{k \mid k}
$$

thus, $\epsilon_{k}$ is the squared estimation error $\tilde{\mathbf{e}}_{k \mid k}$ which is normalized with its associated covariance $\mathbf{P}_{k \mid k}^{-1}$. Under the assumption that the estimation error is approximately Gaussian distributed and the filter is consistent, $\epsilon_{k}$ is $\chi^{2}$ distributed with $n_{e}$ degrees of freedom, where $n_{e}$ is the dimension of $\mathbf{e}: \epsilon_{k} \sim \chi_{n_{e}}^{2}$. Then:

$$
\mathbb{E}\left[\epsilon_{k}\right]=n_{e}
$$

The test will be based on the results of $N$ Monte Carlo Simulations that provide $N$ independent samples $\epsilon_{k}^{i}, i=1, \ldots, N$, of the random variable $\epsilon_{k}$. The sample average of these $N$ samples is

$$
\bar{\epsilon}_{k}=\frac{1}{N} \sum_{i=1}^{N} \epsilon_{k}^{i} .
$$

If the filter is consistent, $N \bar{\epsilon}_{k}$ will have a $\chi^{2}$ density with $N n_{e}$ degrees of freedom.

Hypothesis $H_{0}$, that the state estimation errors are consistent with the filter calculated covariances is accepted if $\bar{\epsilon}_{k} \in\left[a_{1}, a_{2}\right]$, where the acceptance interval is determined such that:

$$
P\left\{\bar{\epsilon}_{k} \in\left[a_{1}, a_{2}\right] \mid H_{0}\right\}=1-\alpha .
$$

In this chapter, we apply the $95 \%$ probability concentration region for $\bar{\epsilon}_{k}$, i.e. $\alpha$ is 0,05 .

In the TDOA scenario of a stationary emitter, the dimension $n_{e}$ of the emitter is 2 , so the number of degrees of freedom for the NEES is equal to 2 . Basis of the test are the results of $N=1000$ Monte Carlo simulations, we get a total of 2000 degrees of freedom. The interval $[1.878,2.126]$ is obtained for 2000 degrees of freedom with the values of the $\chi^{2}$ table as twosided acceptance interval.

\section{A.3 Estimation algorithms overview}

Powerful estimation algortihms must be found that effectively estimates the emitter state from the noisy measurements. Due to the fact that for passive emitter tracking, measurements and states are not linearly related, only nonlinear methods can be applied. We concentrate on some representatives of the number on nonlinear estimation methods with a focus on the Gaussian Mixture filter which shows good performance in nonlinear measurement situations, (Mušicki et al., 2010). 


\section{A.3.1 MLE}

The MLE is a direct search method and computes at each time step the optimal emitter state based on the complete measurement dataset. It stores the complete measurement dataset and belongs to the batch algorithms. The MLE provides that value of $\mathbf{e}_{k}$ which maximizes the Likelihood function, the conditional probability density function, (30). This means that the MLE minimizes the quadratic form:

$$
g\left(\mathbf{e}_{k}\right)=\sum_{i=1}^{k}\left(z_{i}-h\left(\mathbf{e}_{i}\right)\right)^{T} \mathbf{R}^{-1}\left(z_{i}-h\left(\mathbf{e}_{i}\right)\right)
$$

with respect to $\mathbf{e}_{k}$. Since there is no closed-form MLE solution for $\mathbf{e}_{k}$ in passive emitter tracking using TDOA, FDOA and AOA, a numerical iterative search algorithm is needed to find the minimum of the quadratic form. Therefore, application of MLE suffers from the same problems as the numerical algorithms. The ML method attains asymptotically the CRLB when properly initialized. One disadvantage of the MLE is the high computational effort in comparison to the Kalman filters.

\section{A.3.2 EKF}

The Extended Kalman filter (EKF) is a recursive Bayesian estimator which approximates the nonlinearities by linearization. The Bayes theorem which expresses the posterior probability density function of the state based on all available measurement information is used to obtain an optimal estimate of the state:

$$
p\left(\mathbf{e}_{k} \mid Z_{k}\right)=\frac{p\left(z_{k} \mid \mathbf{e}_{k}\right) p\left(\mathbf{e}_{k} \mid Z_{k-1}\right)}{p\left(z_{k} \mid Z_{k-1}\right)}
$$

with $p\left(z_{k} \mid Z_{k-1}\right)=\int p\left(z_{k} \mid \mathbf{e}_{k}\right) p\left(\mathbf{e}_{k} \mid Z_{k-1}\right) d \mathbf{e}_{k}$.

The filter consists of two steps, prediction using the dynamic equation and update, using the Bayes theorem to process the incoming measurement. Processing a combination of two measurements is the same as filtering first with one measurement and then processing the result with the other measurement, as shown in (Mušicki et al., 2010).

In passive target tracking using TDOA, angle and FDOA measurements, the nonlinearity is in the measurement equations. Thus, the EKF approximates the measurement equations by its first-order Taylor series expansions. Here, the TDOA and AOA measurement functions are differentiated with respect to the position coordinates and the FDOA measurement function is differentiated with respect to the position and velocity coordinates:

$$
\begin{aligned}
& \widehat{\mathbf{H}}_{k}^{t}=\frac{\left(\mathbf{r}_{k}^{(1)}\right)^{T}}{\left\|\mathbf{r}_{k}^{(1)}\right\|}-\frac{\left(\mathbf{r}_{k}^{(2)}\right)^{T}}{\left\|\mathbf{r}_{k}^{(2)}\right\|} \\
& \widehat{\mathbf{H}}_{k}^{\alpha}=\frac{\left(\begin{array}{c}
y_{k}-y_{k}^{(1)} \\
x_{k}^{(1)}-x_{k}
\end{array}\right)^{T}}{\left\|\mathbf{r}_{k}^{(1)}\right\|^{2}} \\
& \widehat{\mathbf{H}}_{k}^{f}=\left[\begin{array}{c}
\mathbf{D}_{k}^{(1)}-\mathbf{D}_{k}^{(2)} \\
\frac{\mathbf{r}_{k}^{(2)}}{\left\|\mathbf{r}_{k}^{(2)}\right\|}-\frac{\mathbf{r}_{k}^{(1)}}{\left\|\mathbf{r}_{k}^{(1)}\right\|}
\end{array}\right]^{T},
\end{aligned}
$$


where

$$
\mathbf{D}_{k}^{(i)}=\frac{\left(\dot{\mathbf{x}}_{k}^{(i)}-\dot{\mathbf{x}}_{k}\right)-\frac{1}{\left\|\mathbf{r}_{k}^{(i)}\right\|^{2}}\left[\left(\dot{\mathbf{x}}_{k}^{(i)}-\dot{\mathbf{x}}_{k}\right)^{T} \mathbf{r}_{k}^{(i)}\right] \mathbf{r}_{k}^{(i)}}{\left\|\mathbf{r}_{k}^{(i)}\right\|}, \quad i=1,2 .
$$

Then the Kalman filter equations are applied. The EKF is highly sensitive to the initialization and works satisfactorily only if the initial value is near the true target position.

\section{A.3.3 UKF}

The Unscented Kalman Filter (UKF) (see (Julier \& Uhlmann, 2004)) deterministically selects a small number of sigma points. These sigma points are propagated through a nonlinear transformation. Since the nonlinearities in passive target tracking are in the measurement equations, the Unscented Transform (UT) is applied in the update step. In the state space, sample points and their weights are deterministically chosen. They represent mean and covariance of the density. The sample points are propagated through the UT. This produces the sampling points in the measurement space. Furthermore a covariance and a cross covariance is computed. Than the Filter Equations are passed.

Alternatively, the UT can be used to transform measurements in the state space. In this chapter, measurements of the two-dimensional measurement space of TDOA and azimuth measurements and their associated measurement covariances are converted into the Cartesian state space. A position estimate and the associate position coavariance in the Cartesian state space is obtained.

The UT algorithm is very simple and easy to apply, no complex Jacobians must be calculated. The initialization is very important. A proper initialization is substantial for good results.

\section{A.3.4 Gaussian Mixture Filter}

The Gaussian Mixture (GM) Filter overcomes the initialization difficulties and divergence problems of the Kalman filter like EKF and UKF. It is a recursive Bayesian estimator like the Kalman filters which uses the Chapman-Kolmogoroff equation for the prediction step and the Bayes equation for the estimation update. The key idea is to approximate the posterior density $p\left(\mathbf{e}_{k} \mid Z_{k}\right)$ by a weighted sum of Gaussian density functions. Applying Bayes rule, the posterior density can be expressed using the likelihood function $p\left(z_{k} \mid \mathbf{e}_{k}\right)$. Therefore, the main step is to approximate the likelihood function by a GM:

$$
p\left(z_{k} \mid \mathbf{e}_{k}\right) \approx p_{A}\left(z_{k} \mid \mathbf{e}_{k}\right)=\sum_{i=1}^{c_{k}} w_{k}^{i} \mathcal{N}\left(z_{k}^{i} ; \hat{z}_{k \mid k}^{i}, \mathbf{R}_{k \mid k}^{i}\right),
$$

where $w_{k}^{i}$ are the weights such that $\sum_{i=1}^{c_{k}} w_{k}^{i}=1$ and $p_{A}$ is the density of approximation which must not be a probability density i. e. does not necessarily integrate to one.

The posterior density is:

$$
p\left(\mathbf{e}_{k} \mid Z_{k}\right)=\frac{p\left(z_{k} \mid \mathbf{e}_{k}\right) p\left(\mathbf{e}_{k} \mid Z_{k-1}\right)}{\int p\left(z_{k} \mid \mathbf{e}_{k}\right) p\left(\mathbf{e}_{k} \mid Z_{k-1}\right) d \mathbf{e}_{k}},
$$

from which one can see, that multiplying $p\left(z_{k} \mid \mathbf{e}_{k}\right)$ by any constant will not change the posterior density.

The approximation of the likelihood is performed in the state space and can be made as accurate as desirable through the choice of the number of mixture components. The problem is to 
formulate an algorithmic procedure for computation of weights, means and covariances. The number of components can increase exponentially over time.

We describe two types of GM filters, a dynamic GM filter and a static GM filter.

\section{Dynamic GM filter}

The dynamic GM filter represents both the measurement likelihood $p\left(z_{k} \mid \mathbf{e}_{k}\right)$ and the state estimate $p\left(\mathbf{e}_{k} \mid Z_{k}\right)$ in the form of Gaussian mixtures in the state space. The algorithm is initialized by approximating the likelihood function after the first measurement in the state space. This Gaussian Mixture yields a modelling of the state estimate too. New incoming TDOA measurements are converted into a Gaussian mixture in the state space. Each component of the state estimate is updated by each measurement component to produce one component of the updated emitter state estimate pdf $p\left(\mathbf{e}_{k} \mid Z_{k}\right)$. This update process is linear and performed by a standard Kalman filter. The number of emitter state estimate components increases exponentially in time. Therefore, their number must be controlled by techniques of pruning and merging.

For each time step the state estimate is obtained by the mean and the covariance:

$$
\begin{aligned}
\hat{\mathbf{e}}_{k} & =\sum_{g=1}^{S_{k} M_{k}} \xi(g) \hat{\mathbf{e}}_{k \mid k}(g) \\
\mathbf{P}_{k \mid k} & =\sum_{g=1}^{S_{k} M_{k}} \xi(g)\left(\mathbf{P}_{k \mid k}+\hat{\mathbf{e}}_{k \mid k}(g) \hat{\mathbf{e}}_{k \mid k}^{T}(g)\right)-\hat{\mathbf{e}}_{k \mid k} \hat{\mathbf{e}}_{k \mid k}^{T} .
\end{aligned}
$$

The GM filter equations can be applied to all passive emitter tracking situations in this chapter. The measurement likelihoods must be presented by their GM.

\section{Static GM filter}

The static GM filter represents the likelihood function $p\left(z_{1} \mid \mathbf{e}_{1}\right)$ after taking the first measurement. The representation in the state space is used. Using the Bayesian equation this likelihood can be used to present the posterior density $p\left(\mathbf{e}_{1} \mid z_{1}\right)$. The component of the Gaussian Mixture are restricted to the number of the components of this Gaussian Sum representation. For each new incoming measurement an EKF is performed to update the posterior density. The algorithmic procedure for computation of weights $w_{g}$, means $\mathbf{e}_{g}$ and covariances $\mathbf{P}_{g}$ of the GM is the same as in the dynamic case. The first measurement is converted into a Gaussian sum. The computational effort of finding a good initialization point for a single KF is omitted here. An EKF is started for each mean and covariance, the weights are updated with the probabilities $p(z \mid \mathbf{e})$. The filter output is the weighted sum of the individual estimates and covariances:

$$
\begin{aligned}
\hat{\mathbf{e}}_{k} & =\sum_{g=1}^{n} w(g) \hat{\mathbf{e}}_{k \mid k}(g) \\
\mathbf{P}_{k \mid k} & =\sum_{g=1}^{n} w(g)\left(\mathbf{P}_{k \mid k}+\hat{\mathbf{e}}_{k \mid k}(g) \hat{\mathbf{e}}_{k \mid k}^{T}(g)\right)-\hat{\mathbf{e}}_{k \mid k} \hat{\mathbf{e}}_{k \mid k}^{T}
\end{aligned}
$$

where $n$ is the number of Gaussian terms. 


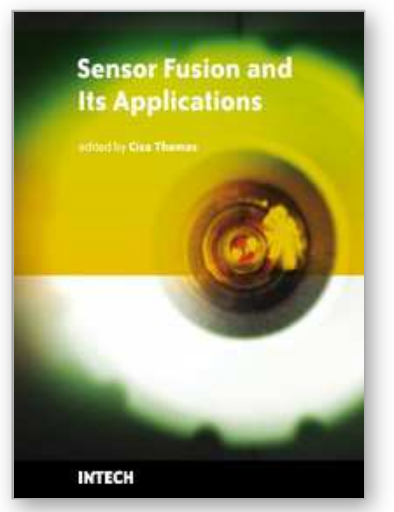

\author{
Sensor Fusion and its Applications \\ Edited by Ciza Thomas
}

ISBN 978-953-307-101-5

Hard cover, 488 pages

Publisher Sciyo

Published online 16, August, 2010

Published in print edition August, 2010

This book aims to explore the latest practices and research works in the area of sensor fusion. The book intends to provide a collection of novel ideas, theories, and solutions related to the research areas in the field of sensor fusion. This book is a unique, comprehensive, and up-to-date resource for sensor fusion systems designers. This book is appropriate for use as an upper division undergraduate or graduate level text book. It should also be of interest to researchers, who need to process and interpret the sensor data in most scientific and engineering fields. The initial chapters in this book provide a general overview of sensor fusion. The later chapters focus mostly on the applications of sensor fusion. Much of this work has been published in refereed journals and conference proceedings and these papers have been modified and edited for content and style. With contributions from the world's leading fusion researchers and academicians, this book has 22 chapters covering the fundamental theory and cutting-edge developments that are driving this field.

\title{
How to reference
}

In order to correctly reference this scholarly work, feel free to copy and paste the following:

Regina Kaune, Darko Musicki and Wolfgang Koch (2010). On Passive Emitter Tracking in Sensor Networks, Sensor Fusion and its Applications, Ciza Thomas (Ed.), ISBN: 978-953-307-101-5, InTech, Available from: http://www.intechopen.com/books/sensor-fusion-and-its-applications/on-passive-emitter-tracking-in-sensornetworks

\section{INTECH}

open science | open minds

\author{
InTech Europe \\ University Campus STeP Ri \\ Slavka Krautzeka 83/A \\ 51000 Rijeka, Croatia \\ Phone: +385 (51) 770447 \\ Fax: +385 (51) 686166 \\ www.intechopen.com
}

\author{
InTech China \\ Unit 405, Office Block, Hotel Equatorial Shanghai \\ No.65, Yan An Road (West), Shanghai, 200040, China \\ 中国上海市延安西路65号上海国际贵都大饭店办公楼 405 单元 \\ Phone: +86-21-62489820 \\ Fax: $+86-21-62489821$
}


(C) 2010 The Author(s). Licensee IntechOpen. This chapter is distributed under the terms of the Creative Commons Attribution-NonCommercialShareAlike-3.0 License, which permits use, distribution and reproduction for non-commercial purposes, provided the original is properly cited and derivative works building on this content are distributed under the same license. 